\title{
Editorial
}

\section{Las mejores universidades peruanas en producción científica 2017}

Los rankings universitarios han ido ganando relevancia en las últimas décadas, al ser cada vez más empleados por los estudiantes y sus familias para la toma de decisiones sobre dónde seguir su educación universitaria, por los académicos para decidir respecto al desarrollo de su carrera profesional, por los funcionarios públicos para la asignación de recursos destinados a la docencia e investigación o por los filántropos para elegir las instituciones y programas académicos a los que apoyar, entre otros grupos de interés en el sistema universitario (Eccles, 2002). Pero hoy este instrumento, siguiendo a $\mathrm{O}^{\prime}$ Connell (2013), también es utilizado por las universidades para la toma de decisiones respecto a estrategias de gestión, planificación e implementación de mejoras e incluso algunas se plantean metas estratégicas de acuerdo con la posición que ocupan en los rankings.

En el Perú, la Superintendencia Nacional de Educación Superior Universitaria (Sunedu) presentó la primera versión del Ranking de Universidades del 2017 basada solo en un componente de evaluación: la producción en investigación científica, no se ha considerado otros aspectos importantes como calidad docente, la infraestructura (aulas, laboratorios implementados, bibliotecas, auditorios, áreas de deportes y recreación, tecnologías de la información y comunicación implementadas, etc); los convenios de cooperación con otras instituciones académicas en camino a la internacionalización, la calidad de empleo de los egresados, las acciones de responsabilidad social universitaria, entre otros.

La fuente de información utilizada por la Sunedu para este ranking corresponde al Incites Bencharking \& Analytics, que actualiza cada dos meses los registros de las publicaciones en la Web of Science Core Collection, una de las matrices de información bibliográfica más importantes del mundo. Las mediciones fueron de publicaciones académicas que a partir de índices de citación -instrumentos que identifican las citas existentes entre dichas publicaciones- buscan medir el impacto de la investigación realizada (Sunedu, 2017). El impacto, según Adam (2002) se puede medir a nivel de artículo de revista, investigador, grupo de investigación, institución e incluso territorio. Los indicadores seleccionados para medir la producción científica fueron cuatro: el número de artículos originales publicados por la universidad en revistas indizadas en Web of Science Core Collection, período
2014-2016; el número de documentos citables producidos en la esta web durante el mismo período; el índice $\mathrm{H}$ de la universidad en la referida web entre 1996 y 2016; y el número de artículos que están incluidos en el $10 \%$ de los trabajos más citados en dicha base de datos, por áreas de estudio y año de publicación a nivel mundial, dentro de la ventana de tiempo 2014-2016 (Sunedu, 2017).

Así, de un total de 132 universidades, las diez primeras ubicaciones del ranking de investigación 2017, elaborado por Sunedu, están ocupadas en el país por 1) Pontificia Universidad Católica del Perú (100 puntos), 2) Universidad Peruana Cayetano Heredia $(69,187), 3)$ Universidad Nacional Mayor de San Marcos $(54,548)$, 4) Universidad Nacional Agraria La Molina $(38,432), 5)$ Universidad Nacional de Ingeniería $(26,086), 6)$ Universidad Nacional San Antonio Abad del Cusco $(23,584), 7)$ Universidad Nacional de Trujillo $(20,045), 8)$ Universidad Científica del Sur $(19,865)$, 9) Universidad de Piura $(14,843)$ y 10) Universidad del Pacífico $(11,088)$.

Este ranking general de investigación fue elaborado en seis áreas del conocimiento: Ciencias Naturales, Ingeniería y Tecnología, Medicina y Ciencias de la Salud, Ciencias Agrícolas, Ciencias Sociales y Humanidades. El trabajo generó un ranking por áreas. En Ciencias Naturales lideran Pontificia Universidad Católica del Perú (PUCP) y luego la Universidad Peruana Cayetano Heredia; en Ingeniería y Tecnología, la Pontificia Universidad Católica del Perú y la Universidad Nacional de Ingeniería; en Medicina y Ciencias de la Salud, la Universidad Peruana Cayetano Heredia y la Universidad Nacional Mayor de San Marcos; en Ciencias Agrícolas, la Universidad Nacional Agraria La Molina y la Universidad Nacional Mayor de San Marcos; en Ciencias Sociales, la Pontificia Universidad Católica del Perú y la Universidad Peruana Cayetano Heredia; y en Humanidades, la Pontificia Universidad Católica del Perú y la Universidad Nacional de Trujillo (Sunedu, 2017). La PUCP lidera no solo el ranking general sino también el ranking por áreas, tiene la mayor calificación en cuatro de las seis áreas. Las otras dos áreas están lideradas por la Universidad Peruana Cayetano Heredia (Medicina y Ciencias de la Salud) y la Universidad Nacional Agraria La Molina (Ciencias Agrícolas). 
La revista América Economía, que por varios años consecutivos elabora el ranking de universidades, el 2017 evaluó a 25 universidades que voluntariamente participaron. En su metodología consideran la medición de ocho dimensiones: calidad docente $(25$ $\%)$, investigación e innovación (25\%), empleabilidad (15\%), acreditación (10\%), internacionalización (10 $\%)$, infraestructura (5\%), selectividad (5\%) e inclusión (5\%).

El componente de investigación e innovación del ranking de América Economía, que representa el 25 $\%$ de las dimensiones de evaluación, difiere del mismo componente desarrollado por la Sunedu, le da $60 \%$ a la producción científica, $20 \%$ a los fondos concursables de investigación, 7,5\% al presupuesto en investigación, $7,5 \%$ al número de investigadores y $5 \%$ a las patentes industriales. En la producción científica mide en un $70 \%$ los papers producidos (cantidad trianual de papers indizados en un $30 \%$ respectivamente en ISI, SCIELO, Scopus y $10 \%$ en otras bases de datos, con filiación explícita a la universidad) y en un 30 $\%$ la productividad (tasa que relaciona la cantidad de papers y la cantidad de profesores a jornada completa); en fondos concursales de investigación considera el monto bruto anual adjudicado por la universidad ante organismos estatales, privados 0 multilaterales; en presupuesto en investigación evalúa el porcentaje anual de recursos de la universidad a este rubro; en número de investigadores contabiliza la cantidad de investigadores financiados por Concytec, financiamiento propio, financiamiento externo; y en patentes industriales califica las registradas en Indecopi (Alcántara y Almeida, 2017).

En los diez primeros lugares del ranking 2017 de América Economía, en investigación e innovación, están 1) Universidad Peruana Cayetano Heredia (100 puntos), 2) Pontificia Universidad Católica del Perú $(99,4), 3)$ Universidad Nacional Mayor de San Marcos $(87,4), 4)$ Universidad del Pacífico $(65,2), 5)$ Universidad Peruana de Ciencias Aplicadas (65), 6) Universidad Nacional Agraria La Molina (62), 7) Universidad Católica San Pablo $(54,4), 8)$ Universidad Nacional de Ingeniería (51), 9) Universidad ESAN $(49,7)$ y 10$)$ Universidad Científica del Sur $(46,5)$.

Los dos rankings aún muestran limitaciones metodológicas de medición de la calidad de la investigación en las universidades peruanas, por lo que sus resultados son apenas una aproximación a esta realidad. El ranking de la Sunedu tiene el sesgo en la medición enfocada solo en publicaciones en la base de datos Web of Science Core Collection, lo que no significa necesariamente que hay ausencia de investigación publicada en otras bases de datos de literatura científica de las universidades; su fortaleza, sin embargo, es que la medición se ha realizado sobre el total de la población.

En el caso del ranking de América Economía, su debilidad es que la medición se realiza solo entre las universidades que participan de manera voluntaria, no se mide a todas las universidades, además que la muestra es poco representativa. Su gran fortaleza es que los indicadores o criterios utilizados están mejor definidos, es el caso de la puntuación que otorgan a la publicación científica en las bases de datos Web of Science (WoS), Scopus y SCIELO, todas importantes y en las cuales están las investigaciones de las universidades peruanas.

\section{Referencias bibliográficas}

Adam, D. (2002). Citation analysis: The counting house. Nature, 415, 726-429. doi:10.1038/415726a

Alcántara, C., \& Almeida, A. (17 de Octubre de 2017). América Economía. Obtenido de Descubre los resultados del Ranking de Universidades Peruanas 2017.: https://mba.americaeconomia.com/ articulos/reportajes/descubre-los-resultados-delranking-de-universidades-peruanas-2017

Eccles, C. (2002). The Use of University Rankings in the United Kingdom. United Kingdom: Higher Education in Europe.

O'Connell, C. (2014). Investigating the Role of Global University Rankings in Higher Education Policy Discourse in England. Lancaster: Lancaster University.

SUNEDU. (2017). Informe bienal sobre la realidad universitaria peruana. Lima: SUNEDU.

Wilfredo Bulege Gutiérrez

Revista Apuntes de Ciencia \& Sociedad

Editor en Jefe

wbulege@continental.edu.pe 